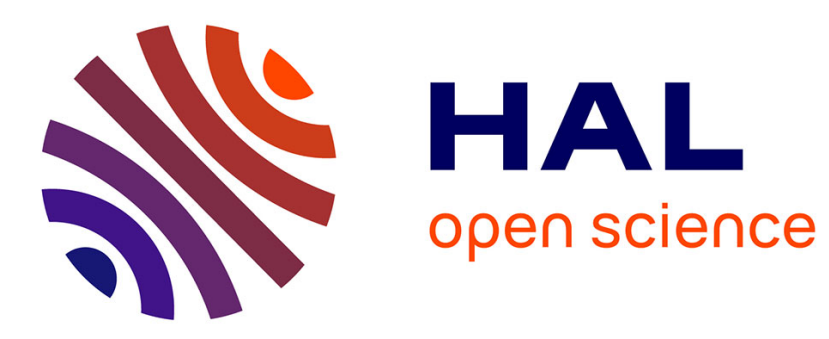

\title{
Essais d'acclimatation du quinquina en Indochine. Quatrième note
}

Alexandre Yersin, André Lambert

\section{To cite this version:}

Alexandre Yersin, André Lambert. Essais d'acclimatation du quinquina en Indochine. Quatrième note. Revue de botanique appliquée et d'agriculture coloniale, 1931, 11 (117), pp.301-304. pasteur00538674

\section{HAL Id: pasteur-00538674}

\section{https://hal-pasteur.archives-ouvertes.fr/pasteur-00538674}

Submitted on 23 Nov 2010

HAL is a multi-disciplinary open access archive for the deposit and dissemination of scientific research documents, whether they are published or not. The documents may come from teaching and research institutions in France or abroad, or from public or private research centers.
L'archive ouverte pluridisciplinaire HAL, est destinée au dépôt et à la diffusion de documents scientifiques de niveau recherche, publiés ou non, émanant des établissements d'enseignement et de recherche français ou étrangers, des laboratoires publics ou privés. 


\section{REVUE \\ de \\ Botanique Appliquée \\ et \\ D'AGRICULTURE TROPICALE}

Publiée sous la Direction du

\section{Prof. Aug. CHEVALIER}

Professeur au Muséum national d Histoire naturelle, Chef de la Mission permanente d'Etudes des Cultures et Jardins d'Essats coloniaux,

Secrétaire général de lAssociation « Colonies-Sciences n.

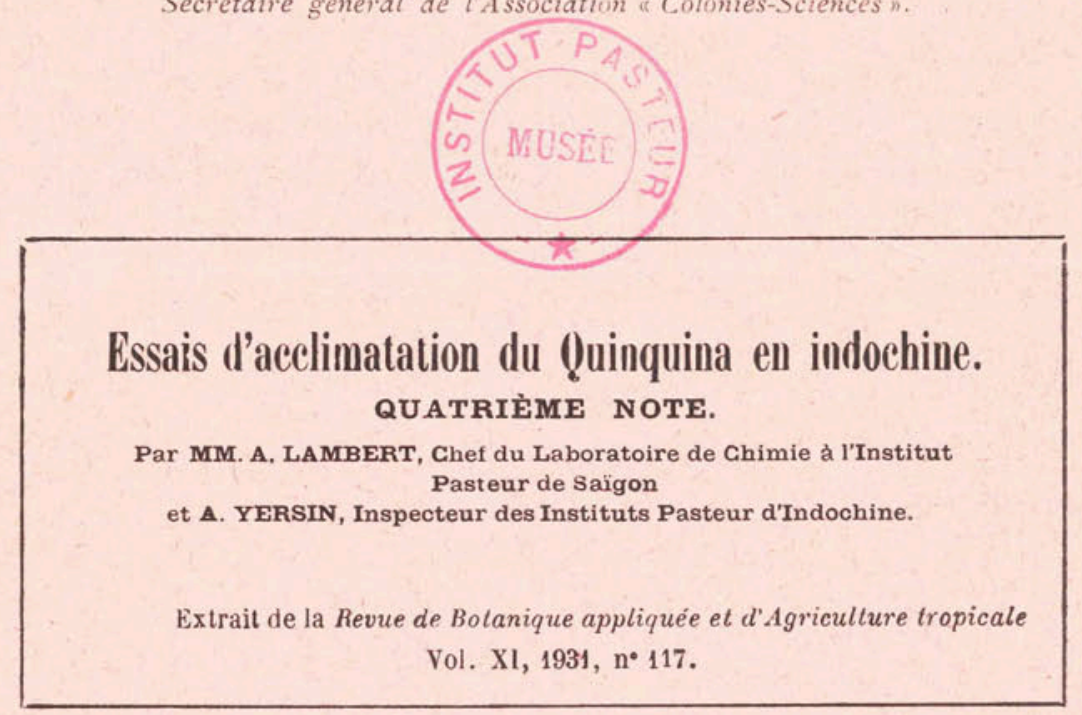

PARIS

Laboratoire d'Agronomie coloniale

Au Muséum, 57, rue Guvier 


\title{
24.769
}

\section{Essais d'acclimatation du 0uinquina en Indochine.}

\section{QUATRIËME NOTE}

\author{
Par MM. A. LAMBERT, Ghef du Laboratoire de Ghimie à l'Institut \\ Pasteur de Saïgon
}

et A. YERSIN, Inspecteur des Instituts Pasteur d'Indoohine.

L'Institut Pasteur poursuit, depuis 1917, des essais d'acclimatation des arbres à Quinquina en Indo-Chine.

Les résultats obtenus ont fait l'objet de trois notes parues dans cetle revue (1).

La quatrième note, que nous présentons aujourd'hui, a pour but de relater brièvement le développement de nos stations d'essai pendant l'année 1929.

Station de Dran. - Au cours de l'année 1929, les arbres plantés à Dran ont continué à se développer vigoureusement.

Les mensurations effectuées en diverses parcelles ont donné les résultats suivants :

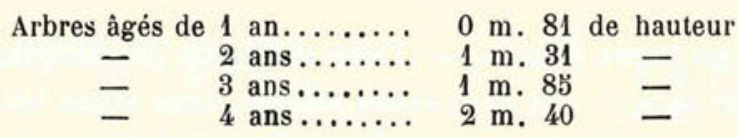

Soit : $0 \mathrm{~m} .50$ environ de croissance annuelle en hauteur,

Essai $n^{\circ} 1$. - Une superficie complantée de 200 Ledgeriana, âgés de 2 ans en 1927, a été amendée à deux reprises, en 1927 et en 1928, au moyen de rejets d'une Légumineuse, le Crotalaria anagyroides, provenant d'une culture d'essai. Ces rejets unt été coupés puis enfouis dans le sol entre les rangées de Cinchona Ledgeriana.

(1) Cf. R. B.A.,t. VII, 1927, p. 2כ0-254, 332-338, 809-816 et t.IX, 1929, p. 507-כั11. Revue de Bot. Appl. 
Le reste de la parcelle a été réservé comme témoin (Ledgeriana du même âge en terre naturelle).

En janvier 1929, vingt arbres de chaque catégorie ont été coupés, afin de déterminer le poids total des écorces par arbre et la teneur moyenne en sulfate de quinine.

Les résultats obtenus ont été les suivants :

\begin{tabular}{|c|c|c|c|}
\hline \multirow{2}{*}{ Nature du terrain } & \multicolumn{3}{|c|}{ (Arbres âgés de 4 ans). } \\
\hline & $\begin{array}{c}\begin{array}{c}\text { Poids d'écorces } \\
\text { par arbre }\end{array} \\
\end{array}$ & $\begin{array}{c}\text { Teneur en sulfate } \\
\text { de quinine } \%\end{array}$ & $\begin{array}{l}\text { sulf. de Qn. } \\
\text { par arbre (gran.. }\end{array}$ \\
\hline & 0 k. 225 & 6,18 & 13,93 \\
\hline $\begin{array}{l}\text { Terre amendée par enfouis } \\
\text { sures de Légumineuses. }\end{array}$ & 0 k. 300 & 6,38 & 19,18 \\
\hline
\end{tabular}

L'influence favorable de la fumure parait évidente.

Essai $n^{\circ}$ 2. - Dans une parcelle voisine, d'une superficie de 0 ha. 3ə̆0, plantée en 192כ, une culture intercalaire de $C$. anagyroides a été entreprise.

En 1929, des élagages ont été pratiqués, les pieds avaient 4 ans d’âge. La teneur des écorces en sulfate de quinine a été reconnue de $6,07 \%$. Dans une parcelle témoin voisine, cette teneur était de $5,67 \%$.

Les résultats obtenus dans l'essai $n^{\circ} 1$ sont donc confirmés par l'essai $n^{\bullet} 2$.

M. F. MaIN, Ingénieur agronome, dans un article paru dans la Revue de Botanique appliquée (t. VIII 1928; page 23), a critiqué, avec quelque raison, nos premiers essais de 1926, sur l'influence de divers engrais sur la croissance des Quinquinas.

Nous avons repris ces expériences en 1928, en cherchant à tenir compte des observations qui nous avaient été présentées.

Les essais sont en cours, mais il ne nous est pas encore possible, aujourd'hui, d'en donner les résultals.

\section{Essais en terre jaune. - (Terre d'origine granitique).}

Dans la $2^{e}$ note (1) que nous avons publiée, nous avons fait mention d'un essai comparatif que nous voulions effectuer à Dran en terre brun chocolat d'origine basaltique, et en terre jaune d'origine granitique.

Nous voulions nous assurer que la belle venue des Quinquinas, sur le plateau de $1000 \mathrm{~m}$. d'altitude, provient principalement des qualités du sol.

Le résultat a été exactement celui que nous avions prévu. Les Ledgeriana repiqués en terre jaune ne se sont pas développés; la

(1) R. B. A. t. VII, 1927, p. 809-816. 
presque totalité a aujourd'hui disparu malgré une culture intercalaire de Légumineuses, que nous avions entreprise pour essayer d'améliorer le sol. Les sujets de même âge, plantés en terre basaltique dans le voisinage immédiat sont superbes et se développent régulièrement.

Essai sur le plateau du petit Lang Bian. - Ces essais, annoncés également dans la $2^{e}$ note, ont été exécutés et poursuivis assidument.

Les premières constatations nous permettent d'établir les points suivants :

A $1600 \mathrm{~m}$. d'altitude, en terre basaltique, la germination est beaucoup plus lente que sur le plateau de $1000 \mathrm{~m}$.

Des pieds de Dran, âgés d'un an, repiqués sur notre terraio d'expérience du Petit Lang-Bian ont bien repris et se sont vigoureusement développés, mais leur croissance est notablement plus lente que celle des témoins de Dran.

Au mois de mai 1929, ces pieds, après 22 mois de repiquage, alteignaient une taille moyenne de $0 \mathrm{~m}$. 89. Les pieds contemporains de Dran avaient 1,20 .

Les arbres du plateau du Petit Lang-Bian ont commencé à fleurir deux ans après le repiquage, en même temps que les pieds de même âge à Dran et à Djiring.

Il ne semble donc pas que la floraison précoce, observée à Dran et à Djiring, soit dûe à un défaut d'altitude de ces stations, comme nous l'avions supposé.

Station de Djiring. - Le développement des arbres à Djiring a été le suivant :

$$
\begin{array}{cccc}
\text { Arbres âgés de } 1 \text { an } \ldots \ldots \ldots . & 0 \mathrm{~m} .64 & \\
- & \text { de } 2 \text { ans........ } & 1 \mathrm{~m} .29 & 0 \mathrm{~m} .65 \\
- & \text { de } 3 \text { ans........ } & 2 \mathrm{~m} .07 & 0 \mathrm{~m} .78
\end{array}
$$

La croissance paraît être, à Djiring, plus rapide qu'à Dran, probablement parce que le terrain y est meilleur.

Nous avons continué les prélèvements systématiques sur les Ledgeriana âgís de 4 à 5 ans, cultivés en terre naturelle.

Le tableau ci-contre donne les chiffres comparatifs des essais sur 10 Ledgeriana àgés de 4 ans en 1928 et sur 10 Ledgeriana du même lot, donc âgés de ơ ans, en 1929 : 




L'augmentation du pourcentage en sulfate de quinine a été très notable au cours de cette période. Le poids des écorces a augmenté de $9 \%$ et le poids de sulfate de quinine de $33 \%$.

Pour les prises d'échantillons, les arbres sont coupés à $10 \mathrm{~cm}$. environ au dessus du sol et soigneusement numérotés.

Presque toujours, dans la suite, des rejets vigoureux se développent, qui permettent de conserver les sujets.

Nous avons constaté que ces rejets fleurissent deux ans après leur apparition.

Nous avons essayé de protéger, au moyen d'une moustiquaire, la floraison d'un de ces arbres à rejets de la station de Dran. La floraison a été assez abondante, mais très peu de fruits se sont formés et les graines retirées de ces fruits ont peu ou pas germé. Il semblerait donc, à moins que ce cas soit exceptionnel, qu'une certaine hybridation est nécessaire pour l'obtention de graines fertiles.

L'analyse des écorces des rejets âgés de 2 et 3 ans a donné une proportion de sulfate de quinine sensiblement égale à celle des branches du même âge.

Voici, par exemple, ce que nous avons observé pour les Dankia de Dran, coupés à $10 \mathrm{~cm}$. au-dessus du sol en 1927 et dont les rejets ont été coupés et analysés en $\mathbf{1 9 3 0 .}$

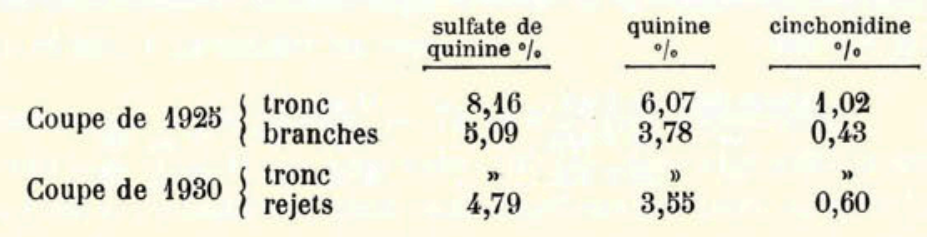

Cette expérience tend à démontrer qu'il n'y a aucun intérêt à envisager une exploitation de Quinquinas consistant à couper les arbres au lieu de les arracher, dans l'espoir de pouvoir utiliser, dans la suite, les rejets sans avoir à faire une plantation nouvelle.

Avec cette façon d'opérer, on se priverait du fort pourcentage de l'écorce du tronc et l'on n'obtiendrait qu'une plantation composée de rejets à basse teneur en sulfate de quinine. 